\title{
Clinical features and influencing prognostic factors in patients with anti-NMDAR encephalitis: a cohort follow-up study in Chinese patients
}

\section{Yingxin Wang}

Nanjing Brain Hospital

Ailiang Miao

Nanjing Brain Hospital

Yongwei Shi

taizhou fourth people's hospital

Jianqing Ge

Nanjing Brain Hospital

Lingling Wang

Nanjing Brain Hospital

Haiyan Xu

Nanjing Brain Hospital

Chuanyong Yu

Nanjing Brain Hospital

Yuanwen Yu

Nanjing Brain Hospital

Shuyang Huang

Nanjing Brain Hospital

Wenwen Jiang

Nanjing Brain Hospital

Yihan Li

Nanjing Brain Hospital

Xiaoshan Wang ( $\nabla$ xiaoshanwang1971@163.com)

\section{Research}

Keywords: Anti-N-methyl-d-aspartate receptor encephalitis, clinical symptoms, cerebrospinal fluid antibody titers, electroencephalography, prognosis

Posted Date: November 22nd, 2019

DOI: https://doi.org/10.21203/rs.2.17708/v1 
License: (c) (i) This work is licensed under a Creative Commons Attribution 4.0 International License. Read Full License 


\section{Abstract}

Objectives The clinical manifestations of patients with anti-N-methyl-D-aspartate receptor (anti-NMDAR) encephalitis in East China and factors associated with prognosis were analyzed.

Methods A retrospective study of 106 patients (58 females; 48 males) with anti-NMDAR encephalitis in East China was carried out from June 2015 to February 2019. Clinical features and factors influencing outcomes were reviewed.

Results Behavioral changes were observed in $74.5 \%$ (79/106) of patients, and comprised the initial symptoms in $61.3 \%(65 / 106)$. Seizures were observed in $67 \%(71 / 106)$ of patients, and served as initial symptoms in $31.1 \%$ (33/106). A total of $54.9 \%$ (39/71) of seizures were focal seizures. More clinical symptoms were observed in female patients than in male patients $(P=0.000)$. Similarly, background activity (BA) with cerebrospinal fluid (CSF) antibody titers at the peak stage was more severe in female patients than in male patients $(P=0.000)$. The Binary logistic regression and ROC curve analyses revealed the factors associated with poor outcomes included consciousness disturbance (OR: 4.907, 95\% Cl: 1.653-14.562, $P=0.004$; area: $65.4 \%$, sensitivity: $44.2 \%$, specificity: $86.5 \%, P=0.014$ ), EEG BA (OR: 3.743, 95\% Cl: 1.766-7.932, $P=0.001$; area: $76.6 \%$, sensitivity: 73\%, specificity: $75 \%, P=0.000$ ), number of symptoms (OR: 2.911, 95\% Cl: 1.811-4.679, $\mathrm{P}=0.000$; area: $77.1 \%$, sensitivity: $59.5 \%$, specificity: $78.6 \%, \mathrm{P}$ $=0.000$ ) and CSF antibody titer (OR: $31.778,95 \% \mathrm{Cl}: 8.891-113.57, \mathrm{P}=0.000$; area: $83.9 \%$, sensitivity: $89.2 \%$, specificity: $78.6 \%, \mathrm{P}=0.000$ ).

Conclusions EEG BA and number of symptoms were associated with CSF antibody titers. Consciousness disturbances, EEG BA, number of symptoms and CSF antibody titers served as predictors of poor outcomes.

\section{Introduction}

Anti-N-methyl-d-aspartate receptor (anti-NMDAR) encephalitis is an autoimmune disease associated with serum antibodies against functional NMDARs $[1,2]$. This syndrome usually develops with a sequential presentation of symptoms, including headache and fever, followed by behavioral changes and psychosis. Seizures can occur at any stage but most commonly manifest early [3]. Magnetic resonance imaging (MRI) remains one of the most important examinations for the diagnosis of central nervous system diseases. As reported, normal brain MRI results have been observed in most patients [3-5].

Electroencephalography (EEG) may be useful for diagnosing anti-NMDAR encephalitis [6-8]. The number of clinical symptoms and electroencephalography (EEG) results were associated with cerebrospinal fluid (CSF) antibody titers [9]. To date, no study has established whether EEG background activity (BA) during the peak stage of anti-NMDAR encephalitis is associated with prognosis. Currently available data are also unclear regarding the associations between CSF antibody titers and outcomes.

The purpose of our study is to provide a summary of the clinical features, and analyze the association between clinical features and prognosis in patients with anti-NMDAR encephalitis. 


\section{Patients And Methods \\ Patients}

A total of 106 patients from the Department of Neurology of Nanjing Brain Hospital were included. The inclusion criteria were as follows: (1) patients who fulfilled the anti-NMDAR encephalitis diagnostic criteria with rapid development (disease course $<3$ months) of one or more symptoms, including mental/behavioral disorders, cognitive impairment, language impairment, consciousness disturbance, epilepsy, involuntary movement, autonomic nervous system dysfunction, or central hypoventilation; and (2) patients with positive anti-NMDAR IgG in CSF with or without positive anti-NMDAR IgG in serum. Patients were excluded if they are diagnosed with other diseases, such as viral encephalitis, brain tumor, metabolic diseases, and drug poisoning [10].

\section{Patient data}

The following patient data were collected: age; sex; presence or absence of malignancy (teratomas and others); neurological symptoms such as seizures and psychiatric symptoms; high (1:10 or 1:32) or low (1:1 or 1:3.2) CSF anti-NMDAR titers; EEG BA including mild diffuse polymorphic slowing (mild DPS), moderate diffuse polymorphic slowing (moderate DPS), severe diffuse polymorphic slowing (SDPS) and extreme delta brush (EDB); MRI; arterial spin labeling (ASL); lumbar puncture; and modified Rankin scale (mRS) scores after 12 to 50 months of immunosuppressive treatment [8]. The initial stage was within 14 days of symptom onset, the peak stage was 14 to 60 days after symptom emergence, the improvement stage was 60 to 180 days after disease onset, and the recovery stage was 180 days after disease onset $[11,12]$.

\section{Statistics}

Data input and statistical analyses were performed using the Statistical Package for Social Sciences (IBM Corporation, Armonk, NY, USA). The Mann-Whitney U test was adopted to compare EEG categorical variables at the peak stage between female and male patients and between patients with low and high CSF antibody titers. The independent-samples $t$ test was used to compare symptoms between female and male patients, and between patients with low and high CSF antibody titers, as well as to compare CSF white cell and protein levels between patients with abnormal T2/ fluid-attenuated inversion recovery (FLAIR) image and patients with normal T2/FLAIR image. The binary logistic regression analysis and receiver operating characteristic $(\mathrm{ROC})$ curve were used to analyze the association between age, sex, consciousness disturbance, CSF antibody titers, EEG BA during peak stage, number of symptoms, imaging results, relapse, intensive care unit (ICU) admissions, follow-up period and prognosis in patients with anti-NMDAR encephalitis. $\mathrm{P}<0.05$ indicated statistical significance.

\section{Results}




\section{Patient characteristics}

We retrospectively identified 106 patients with anti-NMDAR encephalitis (58 females; 48 males). The ages of the male patients were higher than those of the female patients $(36.49 \pm 2.3 v s 25.94 \pm 1.64, P=0.000$; Fig. 1A, Table 1).

\section{Clinical characteristics}

High CSF antibody titers were observed in 60 patients with anti-NMDAR encephalitis and low CSF antibody titers in 37 patients. Ovarian teratomas were observed in 4 patients, and 1 patient manifested pituitary microadenoma. Eight patients experienced relapse. Eleven patients were admitted the ICU.

Behavioral changes were observed in $74.5 \%(79 / 106)$ of the patients. Behavioral changes comprised the initial symptoms in $61.3 \%(65 / 106)$. Seizures were observed in $67 \%(71 / 106)$ of the patients, and $54.9 \%$ $(39 / 71)$ of seizures were focal seizures. Seizures served as initial symptoms in $31.1 \%(33 / 106)$ of the patients. Constant chewing was noted in 10 female patients with high CSF antibody titers during the peak clinical state. The patients with high CSF antibody titers experienced more clinical symptoms than those with low CSF antibody titers (female mean: $4.06 \pm 0.98$ vs $1.88 \pm 0.86$, respectively, $P=0.000$; male mean: $2.29 \pm 1.0$ vs $1.50 \pm 0.79, P=0.006$ ). Similarly, more clinical symptoms were observed in the female patients than in the male patients (mean: $4.06 \pm 0.98$ vs $2.29 \pm 1.0, P=0.000$ ) (Fig. 1B, Table 1).

A total of $72.5 \%$ (74/102) of patients showed normal MRIs. Brain lesions were observed in $27.45 \%$ of patients (28/102). On MRI, hyperintensities involving the hippocampus, temporal cortex, insula, parietal cortex, frontal cortex, occipital lobe, white matter, basal ganglia, thalamus, brainstem, cerebellum, and splenium of the corpus callosum were noted in $11.8 \%$ (12/102), $12.7 \%(13 / 102), 6.9 \%(7 / 102), 7.8 \%$ (8/102), 3.9\% (4/102), 0.98\% (1/102), 3.9\% (4/102), 2.9\% (3/102), 0.98\% (1/102), 0.98\% (1/102), $0.98 \%$ $(1 / 102)$, and $0.98 \%(1 / 19)$ of the patients, respectively (Table 1, Fig.2). During the peak stage of the disease, focal high blood flow with normal MRIs were observed from 56.52\% (13/23) ASL of anti-NMDAR encephalitis patients in Table 1. Focal high blood flow with brain lesions were observed in $39.13 \%(9 / 23)$ ASL of patients (Table 1). CSF white cells in patients with abnormal T2/FLAIR were higher than those in patients with normal T2/FLAIR (mean: $89.63 \pm 29.53$ vs $18.44 \pm 7.42$, respectively, $P=0.000$ ). Similarly, CSF protein levels were higher in patients with abnormal T2/FLAIR than in patients with normal T2/FLAIR (mean: $0.63 \pm 0.06$ vs $0.4 \pm 0.03, P=0.002$ ) (Table 1, Fig. 3).

One hundred and fourteen EEG or VEEG recordings were obtained from 92 patients. EEG BA was significantly aggravated at 12 days (Fig.4 A, C, F and G). A total of 107 EEGs and CSF antibody titers were collected from 85 patients. $91 \mathrm{EEGs}$ and CSF antibody titers during 12 to 60 days from 76 patients were obtained. 76 first EEGs during from 12 to 60 days (median 19.5 days) from 76 patients were analyzed. SDPS was observed in 15 anti-NMDAR encephalitis patients, moderate DPS in 32 patients, mild DPS in 25 patients, and normal activity in 4 patients, separately (Table 1, Fig.4 B, D, F and H). The BA during the peak stage in female patients with high CSF antibody titers was more severe than that in female patients with low CSF antibody titers (Fig.4B VS Fig.4C; Mann-Whitney Utest, $P=0.000$ ). BA in those with high 
CSF antibody titers at the peak stage was more severe in female patients than in male patients (Fig.4B VS Fig.4F; Mann-Whitney Utest, $P=0.000$ ) (Table 1, Fig.4).

\section{Factor-related prognosis}

A total of 103 (97.2\%) patients were treated with first-line immunotherapy, $3(2.8 \%)$ with second-line immunotherapy, and 1 with tumor removal and immunotherapy.

At follow-up after at least 12 months (median: 19 months; range: 12 to 50 months), 37 patients reached a $\mathrm{mRS}$ of $0-2$, and 53 patients had poor outcomes (mRS $\geq 2$ ) (Table 1). The follow-up period for 16 patients was < 12 months. The age, sex, EEG BA, number of symptoms, CSF antibody titers, consciousness disturbance, imaging results, relapse, ICU admission, follow-up period, and prognosis were analyzed with binary logistic regression analysis and ROC curve analysis. In the binary logistic regression analysis, the factors associated with poor outcomes included consciousness disturbance (OR: 4.907, 95\% Cl: 1.65314.562, $P=0.004)$, EEG BA (OR: 3.743, 95\% Cl: 1.766-7.932, $P=0.001)$, number of symptoms (OR: 2.911, 95\% Cl: 1.811-4.679, $P=0.000$ ) and CSF antibody titers (OR: 31.778, 95\% Cl: 8.891-113.57, $P=0.000$ ) (Table 2, Fig. 5). In the ROC curve analysis, predictors for poor outcomes included consciousness disturbance (area: $65.4 \%$, sensitivity: $44.2 \%$, specificity: $86.5 \%, P=0.014$ ), EEG BA (area: $76.6 \%$, sensitivity: 73\%, specificity: 75\%, $P=0.000$ ), number of symptoms (area: $77.1 \%$, sensitivity: $59.5 \%$, specificity: $78.6 \%$, $P=0.000$ ) and CSF antibody titers (area: $83.9 \%$, sensitivity: $89.2 \%$, specificity: $78.6 \%, P=0.000$ ) (Table 2 , Fig. 5).

\section{Discussion}

Our study focused on clinical features and factors related to prognosis in patients with anti-NMDAR encephalitis. The male patients were older than the female patients $(P=0.000)$ (Table 1$)$. There was no sex difference in this sample, which is similar to a previous report on adult-onset anti-NMDAR encephalitis in Korea [13]. The prevalence of teratomas in females over 18 years old was $56 \%$, but only $31 \%$ in females under 18 years old, and only $9 \%$ in females under 14 years old [3]. In short, the younger the female patients, the lower the incidence of teratomas [3]. There were only four female patients with tumors in this study.

Behavioral changes and seizures were the major symptoms. Behavioral changes comprised the initial symptoms in $61.3 \%(65 / 106)$ of the patients with anti-NMDAR encephalitis in this study. Seizures were observed as the onset symptoms in 31.3\% (33/106) of the patients. Moreover, $54.9 \%(39 / 71)$ of the seizures were focal (Table 1). Most patients initially presented with behavioral changes and seizures, which aligned with previous findings $[14,15]$. In an observational cohort study, $87 \%$ of patients exhibited 4 or more categories of symptoms by the end of the first month [16].Our study investigated whether clinical symptoms were associated with sex and CSF antibody titers in patients with anti-NMDAR encephalitis. A greater number of clinical symptoms were noted in patients with high CSF antibody titers than in those with low CSF antibody titers (Table 1, Fig.1), which aligned with our previous findings [9]. The peak-stage BA was worse in the female patients with high CSF antibody titers than in those with low 
CSF antibody titers (Mann-Whitney Utest, $P=0.000$ ) (Table 1, Fig.4). The clinical symptoms were more severe in the female patients with high CSF antibody titers than in male patients with high CSF antibody titers (mean: $3.97 \pm 0.17 v s 2.33 \pm 0.20$, respectively, $P=0.000$ ) (Table 1, Fig.1). Meanwhile, the female patients with high CSF antibody titers showed worse peak stage BA than the male patients with high CSF antibody titers (Mann-Whitney Utest, $P=0.000$ ) (Table 1, Fig.4). The constant chewing observed in the female patients with high CSF antibody titers during the peak stage was unlikely to be caused by temporal or frontal seizures because no epileptiform discharges (EDs) were observed in the EEG recordings, and antiepileptic drugs (AEDs) were ineffective [9]. Constant chewing may be a useful marker of the peak period of the disease.

During the peak stage of the disease, brain lesions were observed in $27.45 \%$ of the patients $(28 / 102)$ in this study. Moreover, 13 patients with normal MRIs showed focal high blood flow in Table 1. In several previous studies, $37.2 \%-50 \%$ of patients showed abnormal brain MR imaging results [3-5]. On MRI, hyperintensities involving the hippocampus, temporal cortex, insula, parietal cortex, frontal cortex, and white matter (frontal region) were noted in $11.8 \%$ (12/102), 12.7\% (13/102), 6.9\% (7/102), 7.8\% (8/102), $3.9 \%(4 / 102)$, and $3.9 \%$ (4/102) of the patients in this study, respectively (Table 1, Fig. 2). In 2017 restingstate functional connectivity in patients with anti-NMDAR encephalitis revealed widespread alterations of functional connectivity correlated with clinical measures [17]. These alterations included impaired hippocampal functional connectivity, decoupling of the medial temporal and the default-mode networks, and an overall impairment in frontotemporal connections. Furthermore, functional connectivity was impaired within distributed large-scale networks, including the sensorimotor, frontoparietal, lateraltemporal, and visual networks. Memory impairment correlated with hippocampal and medial-temporallobe network connectivity, whereas schizophrenia-like symptoms were associated with functional connectivity changes in frontoparietal networks. Machine-learning analyses corroborated these findings and identified frontoparietal and frontotemporal connections as reliably discriminating features between patients and controls, yielding an overall accuracy of $81 \%$ [17]. In a previous study, patients with normal MRIs were younger than patients with abnormal MRIs [18]. There were no statistically significant dfferences in MRI findings (normal or abnormal) between patients with or without tumors, patients experiencing seizures, patients experiencing hypoventilation, or patients experiencing loss of consciousness. Abnormal MRIs did not affect prognosis evaluated by mRS [18]. In this study, CSF white cells in the patients with abnormal T2/FLAIR were higher than those in the patients with normal T2/FLAIR (mean: $89.63 \pm 29.53$ vs $18.44 \pm 7.42$, respectively, $P=0.000$ ). Similarly, there were higher CSF protein levels in patients with abnormal T2/FLAIR than in patients with normal T2/FLAIR (mean: $0.63 \pm 0.06 \mathrm{vs}$ $0.4 \pm 0.03$, respectively, $P=0.002$ ).

In a large unselected cohort of adults, a normal posterior rhythm in the first EEG recording at a median of 19 days from disease onset predicted a favorable clinical outcome, while a severely abnormal EEG was associated with a poor outcome [19]. Ordinal logistic regression showed that the presence of a normal posterior rhythm was associated with lower mRS at final follow-up [19]. Binary logistic regression analysis and ROC curve analysis showed that EEG BA during the peak clinical state (median: 19.5 days) was associated with poor outcomes in this study. The number of symptoms and CSF antibody were also 
predictors of poor outcomes (Table 2, Fig.5). A greater number of clinical symptoms were noted in patients with high CSF antibody titers than in those with low CSF antibody titers (Table 1, Fig. 1). The main epitope targeted by the antibodies is in the extracellular N-terminal domain of the NR1 subunit. Patients' antibodies decreased the numbers of cell-surface NMDA receptors and NMDA-receptor clusters in postsynaptic dendrites, an effect that could be reversed by antibody removal. The severity of antiNMDAR encephalitis was associated with antibody titers [5]. Furthermore, consciousness disturbance was a factor associated with nonfavorable outcomes in this study (Table 2 and Fig. 5), which was similar to a previous study [20]. In previous studies, the predictors of good outcomes were early treatment and lack of ICU admission [4]. In our study, 11 patients were admitted to the ICU. ICU stay was not a predictor of poor outcomes. Many patients are not admitted to the ICU due to the shortage of ICU beds. All patients admitted to the ICU had poor outcomes ( $m R S \geq 2$ ). Relapses were defined as the new onset of symptoms, or worsening of symptoms after at least 2 months of improvement or stabilization. Although relapses were not a predictor of poor outcomes in our study, all patients with relapse had poor outcomes $(m R S \geq 2)$. The follow-up period may have been short in our study. Titulaer et al. followed their antiNMDAR encephalitis patients for a median duration of 24 months and $7.8 \%$ of patients in that study experienced one or more clinical relapses [4].

\section{Conclusion}

EEG BA and the number of symptoms were associated with CSF antibody titers. CSF white cell and protein levels in patients with abnormal T2/FLAIR were higher than in patients with normal T2/FLAIR. Consciousness disturbance, EEG BA, number of symptoms and CSF antibody titers served as predictors of poor outcomes.

\section{Declarations}

\section{Availability of Data and Materials}

Not applicable

\section{Acknowledgements}

We would like to acknowledge the physicians and nurses at Department of Neurology, Nanjing Brain Hospital.

\section{Authors' contributions}

Yingxin Wang and Ailiang Miao: drafting/revising the manuscript and acquire EEG recording. Yongwei Shi: statistical analysis. Xiaoshan Wang: study concept or design and study supervision. Lingling Wang, Jianqing Ge, Yuanwen Yu, Shuyang Huang, Chuanyong Yu, Hongxing Liu, Haiyan Xu and Yihan Li: clinical work. Their contributions helped us to acquire clinical data. 


\section{Funding}

The author(s) disclosed receipt of the following financial support for the research, authorship, and/or publication of this article: The work was supported by the Young Medical Key Talents Foundation of Jiangsu Province (Grant No. QNRC2016053), Training Project for Young Talents of Nanjing Brain Hospital, Young Scientists Fund of the National Natural Science Foundation of China (Grant No. 81501126, http://npd.nsfc.gov.cn/), the National Natural Science Foundation of China (Grant No. 81471324, http://npd.nsfc.gov.cn/), the Fourth Phase of Jiangsu Project 333 Scientific Research Funding Schemes, 2013, the Health Department of Jiangsu Province (Grant No. H201443).

\section{Ethics approval and consent to participate}

Our study was reviewed and approved by the ethical boards of the Affiliated Brain Hospital of Nanjing Medical University, and written informed consent was obtained from the family of all participants.

\section{Consent for publication}

Written informed consent was obtained from all participants

\section{Competing interests}

The author(s) declared no potential conflicts of interest with respect to the research, authorship, and/or publication of this article.

\section{Author details}

${ }^{1}$ Department of Neurology, The Affiliated Brain Hospital of Nanjing Medical University, Nanjing Medical University, Nanjing, Jiangsu, China

2Department of Video-Electroencephalogram, The Affiliated Brain Hospital of Nanjing Medical University, Nanjing Medical University, Nanjing, Jiangsu, China

${ }^{3}$ Medical Records Room, Nanjing Brain Hospital, Nanjing Medical University, Nanjing, Jiangsu, China

${ }^{4}$ Department of Neurology, Taizhou Fourth People's Hospital, Taizhou, Jiangsu, China

\section{Abbreviations}

anti-NMDAR anti-N-methyl-D-aspartate receptor

BA background activity

CSF cerebrospinal fluid

MRI magnetic resonance imaging 
EEG electroencephalography

mild DPS mild diffuse polymorphic slowing

moderate DPS moderate diffuse polymorphic slowing

SDPS severe diffuse polymorphic slowing

EDB extreme delta brush

ASL arterial spin labeling

mRS modified Rankin scale

FLAIR fluid-attenuated inversion recovery

ROC receiver operating characteristic

ICU intensive care unit

\section{References}

1. Dalmau J, Tüzün E, Wu HY, et al. Paraneoplastic anti-N-methyld- aspartate receptor encephalitis associated with ovarian teratoma. Ann Neurol 2007; 61:25-36.

2. Prüss $H$, Dalmau J, Harms $L$, et al. Retrospective analysis of NMDA receptor antibodies in encephalitis of unknown origin. Neurology 2010; 75:1735-1739.

3. Dalmau J, Lancaster E, Martinez-Hernandez E, Rosenfeld MR, Balice-Gordon R. Clinical experience and laboratory investigations in patients with anti-NMDAR encephalitis. Lancet Neurol 2011;10: 6374.

4. Titulaer MJ, McCracken L, Gabilondo I, Armangué T, Glaser C, lizuka T, et al. Treatment and prognostic factors for long-term outcome in patients with anti-NMDA receptor encephalitis: an observational cohort study. Lancet Neurol 2013;12:157-65.

5. Dalmau J, Gleichman AJ, Hughes EG, Rossi JE, Peng X, Lai M, et al. Anti-NMDA-receptor encephalitis: case series and analysis of the effects of antibodies. Lancet Neurol 2008;7: 1091-98.

6. Schmitt SE, Pargeon K, Frechette ES, Hirsch LJ, Dalmau J, Friedman D. Extreme delta brush: a unique EEG pattern in adults with anti-NMDA receptor encephalitis. Neurology 2012; 79 (11): 1094-1100.

7. Foff EP, Taplinger D, Suski J, Lopes MB, Quigg M. EEG findings may serve as a potential biomarker for anti-NMDA receptor encephalitis. Clin EEG Neurosci 2017;48: 48-53.

8. Miao, A., Wang, X. Ictal rhythmic alpha sinusoidal waves in three cases of anti-NMDAR encephalitis. Clin EEG Neurosci 2018; 49: 302-305.

9. Miao A, Du M, Wang L, Ge J, Lu H, Xu H, et al. Analysis of Relation Between Electroclinical Features and Cerebrospinal Fluid Antibody Titers in Patients With anti-NMDAR Encephalitis. Clin EEG Neurosci 
2019;50: 56-62.

10. Graus F, Titulaer MJ, Balu R, Benseler S, Bien CG, Cellucci T, et al. A clinical approach to diagnosis of autoimmune encephalitis. Lancet Neurol 2016;15:391-404.

11. Gitiaux C, Simonnet H, Eisermann M, Leunen D, Dulac O, Nabbout R, et al. Early electro-clinical features may contribute to diagnosis of the anti-NMDA receptor encephalitis in children. Clin Neurophysiol 2013;124: 2354-2361.

12. Nosadini M, Boniver C, Zuliani L, de Palma L, Cainelli E, Battistella PA, et al. Longitudinal electroencephalographic (EEG) findings in pediatric anti-N-methyl-d-aspartate (anti-NMDA) receptor encephalitis: the Padua experience. J Child Neurol 2015;30: 238-245.

13. Lim JA, Lee ST, Jung KH, Kim S, Shin JW, Moon J, et al. Anti-N-methyl-D-aspartate receptor encephalitis in Korea: clinical features, treatment, and outcome. J. Clin Neurol 2014;10: 157-161.

14. Armangue T, Titulaer M, Malaga I, Bataller L, Gabilondo I, Graus F, et al. Pediatric anti-N-methyl-Daspartate receptor encephalitis-clinical analysis and novel findings in a series of 20 patients. $\mathrm{J}$ Pediatr 2013;162: 850-856.

15. Haberlandt E, Ensslen M, Gruber-Sedlmayr U, Plecko B, Brunner-Krainz M, Schimmel M, et al. Epileptic phenotypes, electroclinical features and clinical characteristics in 17 children with anti-NMDAR encephalitis. Eur J Paediatr Neurol 2017;21: 457-4564.

16. Veciana M, Becerra JL, Fossas P, Muriana D, Sansa G, Santamarina E, et al. EEG extreme delta brush: an ictal pattern in patients with anti-NMDA receptor encephalitis. Epilepsy Behav 2015;49: 280-285.

17. Peer M, Prüss H, Ben-Dayan I, Paul F, Arzy S, Finke C. Functional connectivity of large-scale brain networks in patients with anti-NMDA receptor encephalitis: an observational study. Lancet Psychiatry 2017;4: 768-774

18. Wang R, Lai XH, Liu X, Li YJ, Chen C, Li C, et al. Brain magnetic resonance-imaging findings of anti-N-methyl-d-aspartate receptor encephalitis: a cohort follow-up study in Chinese patients. J Neurol 2018;265:362-369.

19. Sonderen AV, Arends S, Tavy DLJ, Bastiaansen AEM, Bruijn MAAM, Schreurs MWJ, et al. Predictive value of electroencephalography in anti-NMDA receptor encephalitis. J Neurol Neurosurg Psychiatry 2018;89: 1101-1106.

20. Aungsumart S, Ha A, Apiwattanakul M. Abnormal level of consciousness predicts outcomes of patients with anti-NMDA encephalitis. J Clin Neurosci 2019;62:184-187.

\section{Tables}

Table 1 Clinical Characteristics of patients with anti-NMDAR encephalitis. 
Sex

Female

Male

Age

Female_age

Male_age

Female_age vs Male_age

a

b

\section{Symptom presentation}

Behavioral changes

Female

a

$\mathrm{b}$

Male

a

b

Seizures

Female

a

$\mathrm{b}$

Male

a

$\mathrm{b}$

Consciousness disturbance

Cognitive impairment

Language impairment

Focal limb weakness

Involuntary movement

Oral
58

48

$25.94 \pm 1.64$

$36.49 \pm 2.3$

Female 36;

Male 24

Female 18;

Male 19

49 (42 OS)

35 (29 OS)

10 (9 OS)

30 (23 OS)

15 (12 OS)

11 (8 OS)

$P=0.000^{\mathrm{d}}$ 


\section{Number of Symptoms}

${ }^{\mathrm{C}}$ Female

${ }^{\mathrm{C}}$ Male

$\mathrm{a}_{\text {Female vs male }}$

$\mathrm{b}_{\text {Female vs male }}$

\section{Imaging}

Normal T2/FLAIR

Abnormal T2/FLAIR

Total ASL

Abnormal T2/Flair and focal high

blood flow

Normal T2/Flair and focal high blood

flow

EEG during peak stage

74

28

23

9

13

Background activity (BA)

Normal

Mild DPS

Moderate DPS

SDPS

EDB

${ }^{\mathrm{C}}$ Female

${ }^{\mathrm{C}}$ Male

$\mathrm{a}_{\text {Female vs male }}$

$\mathrm{b}_{\text {Female vs male }}$

$$
\begin{array}{cc}
4.06 \pm 0.98 \text { vs } 1.88 \pm \\
0.86
\end{array} \quad \begin{gathered}
P=0.000 \\
\mathrm{~d}
\end{gathered}
$$

$\begin{array}{cc}2.29 \pm 1.0 \text { vs } 1.50 & P=0.006 \\ \pm 0.79 & \mathrm{~d}\end{array}$

$4.06 \pm 0.98$ vs

$2.29 \pm 1.0$

$P=0.000$ d

$1.88 \pm 0.86$ vs 1.50 $\pm 0.79$

$P=0.179$

Median 19.5

2 female; 2 male

8 female; 17 male

19 female; 12 male

13 female; 2 male

5 female $^{\text {a }} ; 1$ male
$P=0.000$

e

$P=0.461$

$P=0.000$ e

$P=0.657^{\mathrm{e}}$

CSF:

\section{White cells}

Abnormal T2/FLAIR

Normal T2/FLAIR

$89.63 \pm 29.53$

$18.44 \pm 7.42$

Abnormal T2/FLAIR vs Normal

T2/FlAIR

Normal T2/FLAIR and focal high 
blood flow

\section{Protein}

Abnormal T2/Flair

Normal T2/Flair

Abnormal T2/Flair vs Normal

T2/Flair

\section{ICU admission}

\section{Tumor}

Ovarian teratomas

Other

\section{Therapy}

First-line alone

First-line and second-line

With/without relapse

Female

Male

mRS (follow-up from

$0-1$

12 to 50 months)

$\geq 2$

Std. deviation: standard deviation; mild DPS: mild diffuse polymorphic slowing; moderate DPS: moderate diffuse polymorphic slowing; SDPS: severe diffuse polymorphic slowing; EDB: extreme delta brush; ASL: arterial spin labeling; CSF: cerebrospinal fluid. a High CSF antibody titer (1:10 or 1:32). b Low CSF antibody titer (1:1 or 1:3.2). c Low CSF antibody titer (1:1 or 1:3.2) vs high CSF antibody titer (1:10 or 1:32). $d$ Independent-sample t test; e Mann-Whitney $U$ test. Onset denotes the number of patients with the clinical symptoms manifesting onset symptoms.

Table 2. Factors associated with prognosis in patients with anti-NMDAR encephalitis.

$$
P=\underset{\mathrm{d}}{0.002}
$$

$9^{\mathrm{a}} ; 2^{\mathrm{b}}$

$3^{\mathrm{a}} ; 1^{\mathrm{b}}$

$$
4^{\mathrm{a}}
$$

Median 19

37

53 (4 died) 


\begin{tabular}{|c|c|c|c|c|c|}
\hline \multicolumn{6}{|c|}{ Binary logistic regression analysis } \\
\hline \multirow[t]{2}{*}{ Variables } & $\overline{\mathrm{PRC}}$ & $P$ values & OR values & \multicolumn{2}{|c|}{$95 \% \mathrm{CI}$} \\
\hline & & & & Lower & Upper \\
\hline Consciousness disturbance & 1.591 & 0.004 & 4.907 & 1.653 & 14.562 \\
\hline EEG BA & 1.32 & 0.001 & 3.743 & 1.766 & 7.932 \\
\hline Number of symptoms & 1.068 & 0.000 & 2.911 & 1.811 & 4.679 \\
\hline CSF antibody titers & 3.459 & 0.000 & 31.778 & 8.891 & 113.57 \\
\hline \multirow{2}{*}{\multicolumn{6}{|c|}{ ROC curve }} \\
\hline Variables & & & Sensitivity & \multicolumn{2}{|c|}{ Specificity } \\
\hline Consciousness disturbance & $65.4 \%$ & 0.014 & $44.2 \%$ & \multicolumn{2}{|c|}{$86.5 \%$} \\
\hline EEG BA & $76.6 \%$ & 0.000 & $73 \%$ & \multirow{2}{*}{\multicolumn{2}{|c|}{$\begin{array}{c}75 \% \\
78.6 \%\end{array}$}} \\
\hline Number of symptoms & $77.1 \%$ & 0.000 & $59.5 \%$ & & \\
\hline CSF antibody titers & $83.9 \%$ & 0.000 & $89.2 \%$ & \multicolumn{2}{|c|}{$78.6 \%$} \\
\hline
\end{tabular}

EEG BA: EEG background activity during peak stage; PRC: partial regression coefficient

\section{Figures}



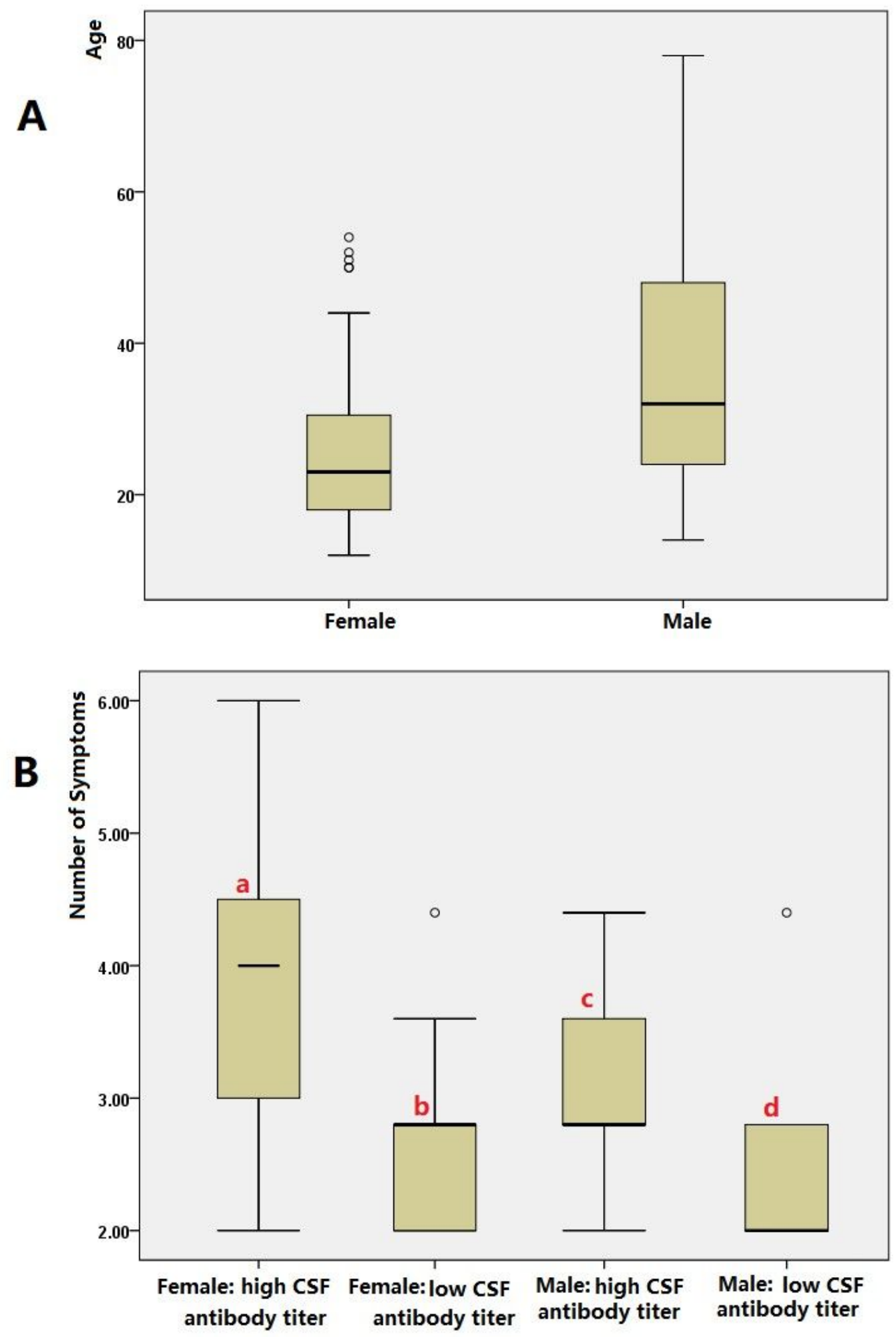

\section{Figure 1}

(A) The male patients were older than the female patients ( $36.49 \pm 2.3$ vs $25.94 \pm 1.64, P=0.000)$. (B) Patients with high CSF antibody titers experienced more clinical symptoms than those with low CSF antibody titers ( $a$ vs b: mean $4.06 \pm 0.98$ vs $1.88 \pm 0.86, P=0.000$; c vs d: mean $2.29 \pm 1.0$ vs $1.50 \pm 0.79$, $\mathrm{P}=0.006$ ). Similarly, more clinical symptoms were observed in the female patients than in the male patients (a vs c: mean $4.06 \pm 0.98$ vs $2.29 \pm 1.0, P=0.000$ ). 

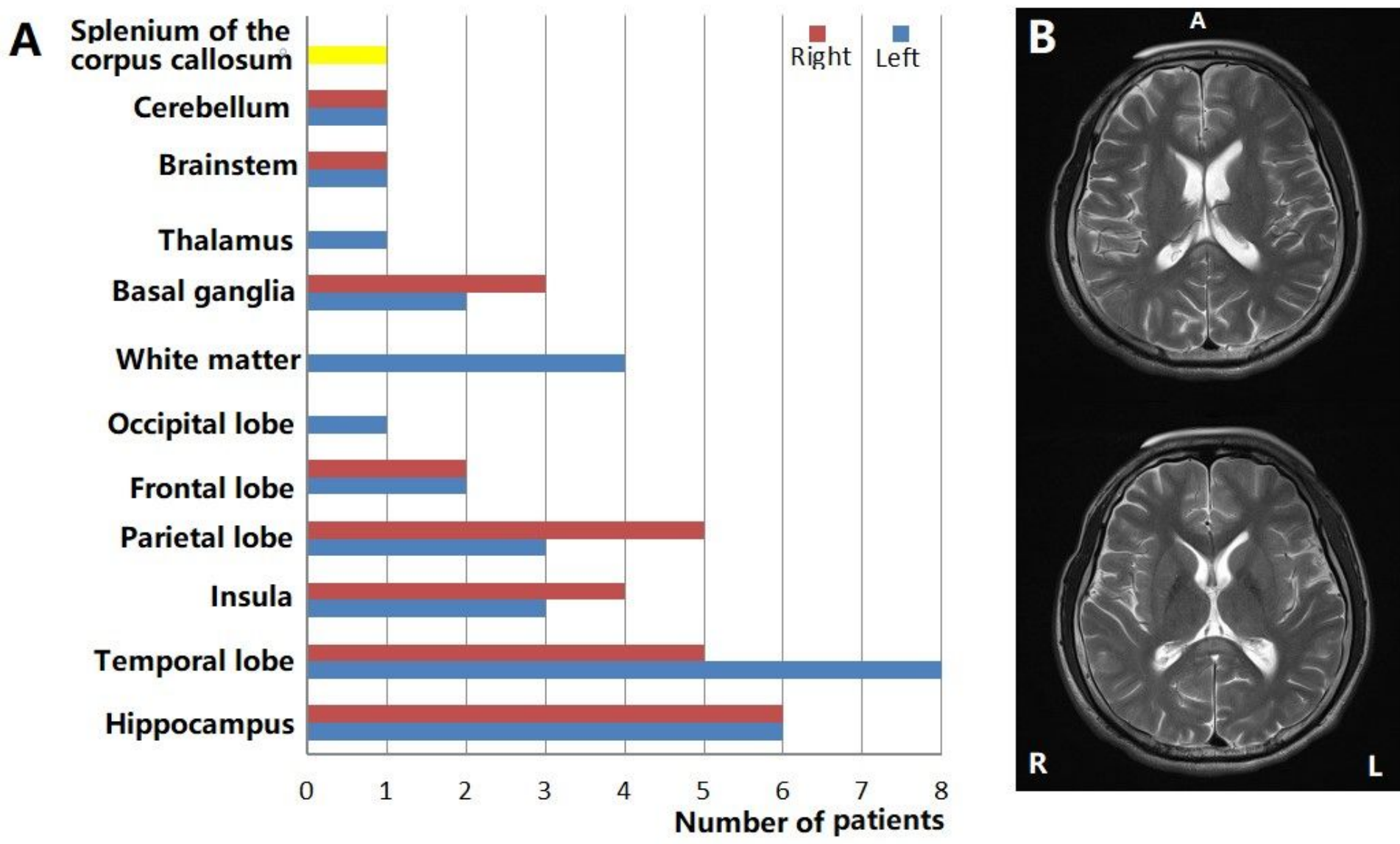

Figure 2

(A) Brain lesions in patients with anti-NMDAR encephalitis. (B) Splenium of the corpus callosum lesions in patient with anti-NMDAR encephalitis. 

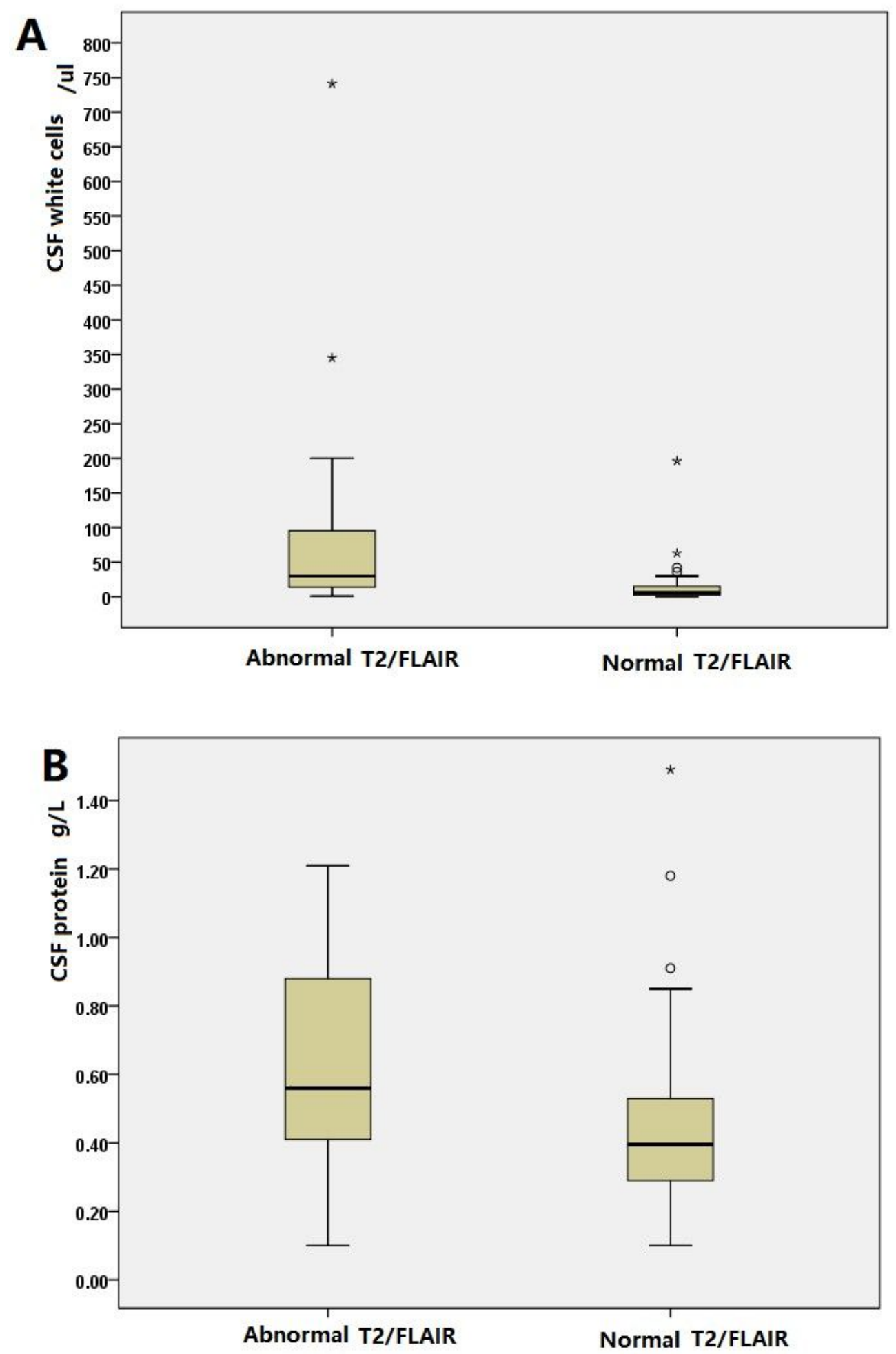

Figure 3

(A) CSF white cells are higher in patients with abnormal T2/FLAIR than in patients with normal T2/FLAIR (mean: $89.63 \pm 29.53$ vs $18.44 \pm 7.42, P=0.000$ ). (B) CSF protein levels are higher in patients with abnormal T2/FLAIR than in patients with normal T2/FLAIR (mean: $0.63 \pm 0.06$ vs $0.4 \pm 0.03, P=0.002$ ). 
Female

high CSF anti-NMDAR titers
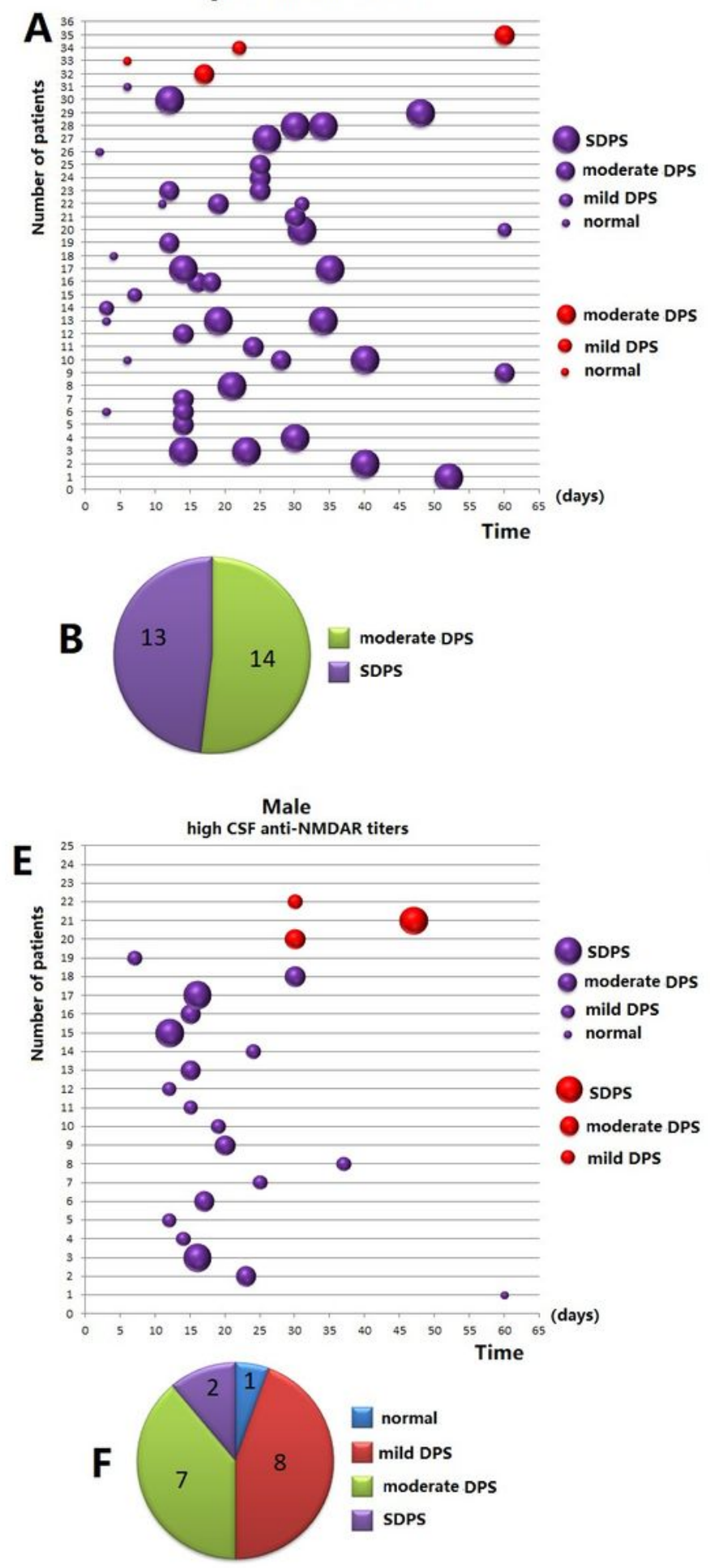

Female
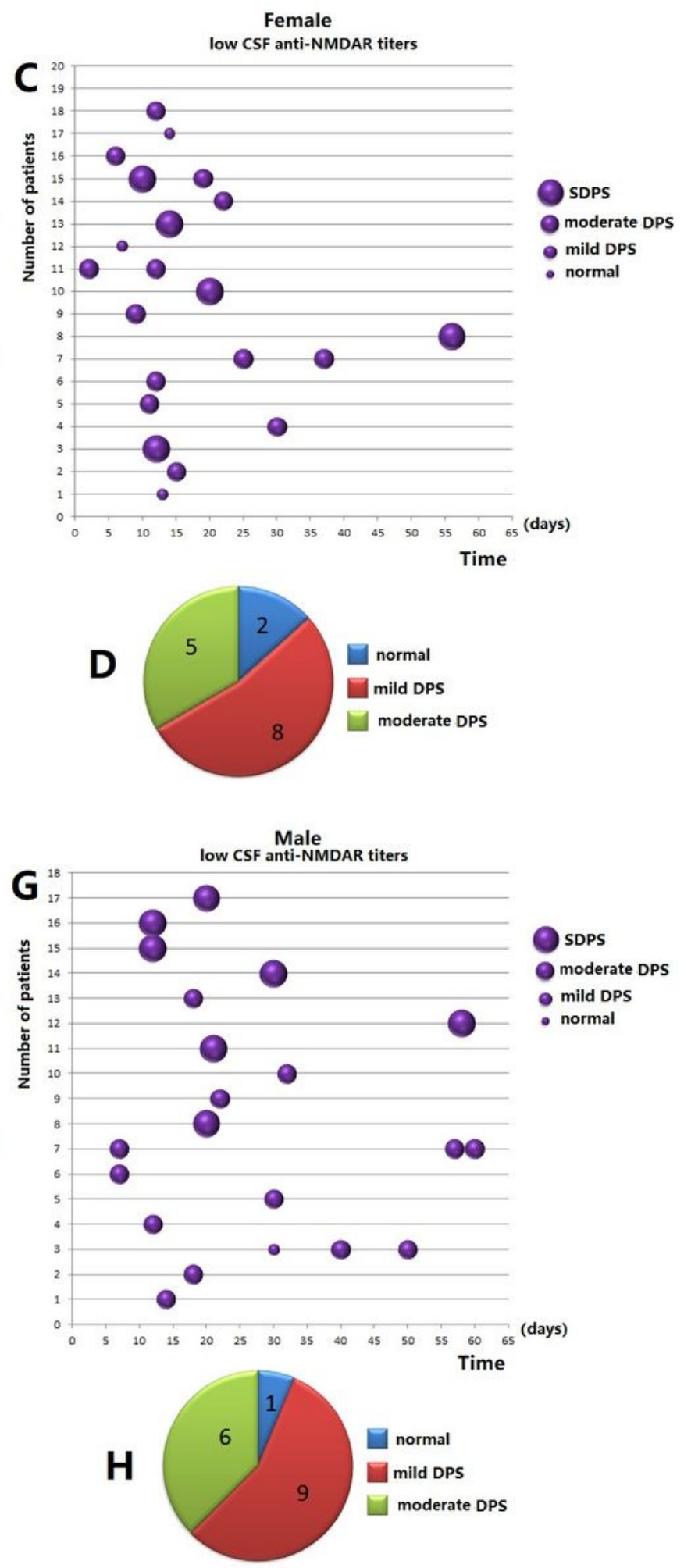

\section{Figure 4}

One hundred and fourteen EEG or VEEG recordings were obtained from 92 patients. EEG BA was significantly aggravated at 12 days (A, C, F and G). A total of 107 EEGs and CSF antibody titers were collected from 85 patients. 76 first EEGs ranging from 12 to 60 days (median 19.5 days) from 76 patients were analyzed. SDPS was observed in 15 anti-NMDAR encephalitis patients; moderate DPS in 32 patients; mild DPS in 25 patients, and normal in 4 patients (Table 1). (B VS D) BA during the peak stage in 
female patients with high CSF antibody titers was more severe than that in patients with low CSF antibody titers (Mann-Whitney U test, $P=0.000$ ). (B VS F) BA with high CSF antibody titers at the peak stage was more severe in female patients than in male patients (Mann-Whitney $U$ test, $P=0.000$ ). Mild DPS: mild diffuse polymorphic slowing; moderate DPS: moderate diffuse polymorphic slowing; SDPS: severe diffuse polymorphic slowing. Purple bubble: patients with EEGs and CSF antibody titers. Red bubble with EEGs and unclear CSF antibody titers: X-axis: time. Y-axis: number of patients.
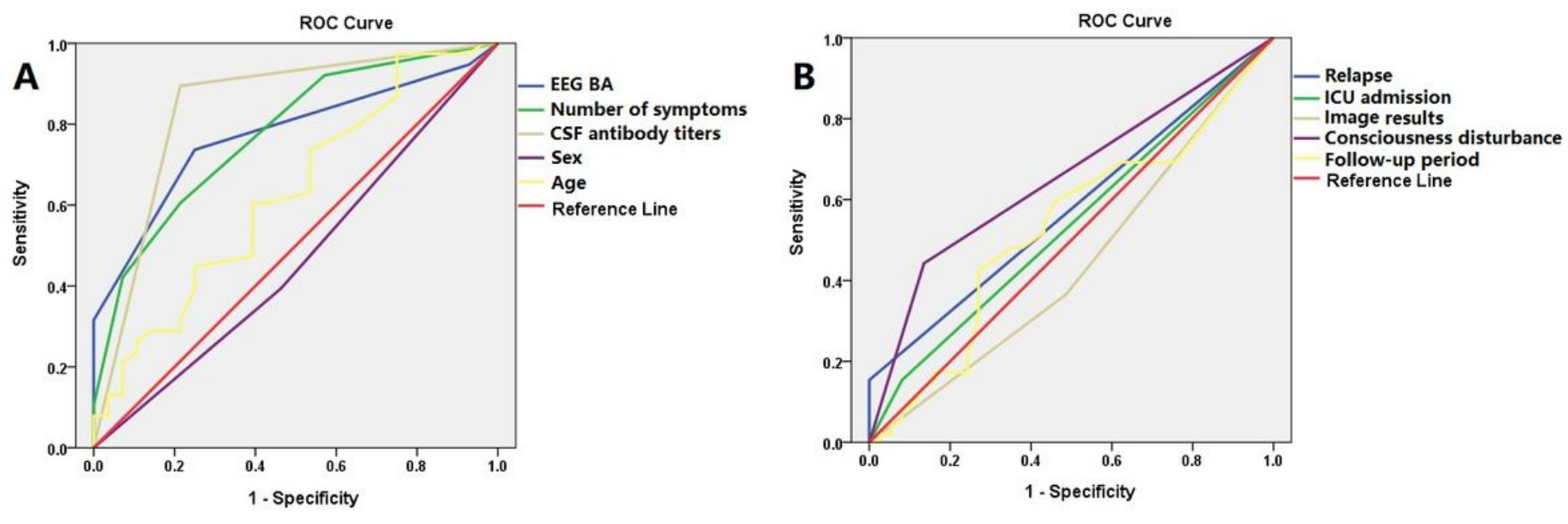

\section{Figure 5}

In the ROC curve analysis, predictors for poor outcomes included consciousness disturbance (area: $65.4 \%$, sensitivity: $44.2 \%$, specificity: $86.5 \%, P=0.014$ ), EEG BA (area: $76.6 \%$, sensitivity: $73 \%$, specificity: $75 \%, P=0.000$ ), number of symptoms (area: $77.1 \%$, sensitivity: $59.5 \%$, specificity: $78.6 \%, P=0.000$ ) and CSF antibody titers (area: $83.9 \%$, sensitivity: $89.2 \%$, specificity: $78.6 \%, P=0.000$ ). BA: background activity. 